\title{
Predicting changes in neuronal excitability type in response to genetic manipulations of $\mathbf{K}^{+}$-channels Marco Arieli Herrera-Valdez*2,3, Sandra D Berger ${ }^{1}$, Carsten Duch ${ }^{1}$ and Sharon Crook ${ }^{1,2}$
}

\author{
Address: ${ }^{1}$ School of Life Sciences, Arizona State University, Tempe, AZ 85287, USA, ${ }^{2}$ Department of Mathematics and Statistics, Arizona State \\ University, Tempe, AZ 85287, USA and ${ }^{3}$ Mathematical, Computational, and Modeling Sciences Center, Arizona State University, Tempe, AZ \\ 85287, USA \\ Email: Marco Arieli Herrera-Valdez* - Marco.Herrera-Valdez.@asu.edu \\ * Corresponding author
}

from Eighteenth Annual Computational Neuroscience Meeting: CNS*2009

Berlin, Germany. 18-23 July 2009

Published: 13 July 2009

BMC Neuroscience 2009, I O(SuppI I):P30 I doi:I0.II86/I47I-2202-I0-SI-P30 I

This abstract is available from: http://www.biomedcentral.com/I47I-2202/I0/SI/P30 I

(c) 2009 Herrera-Valdez et al; licensee BioMed Central Ltd.

The way a neuron undergoes rest-spiking transitions determines the type of excitability and the computational properties of a neuron. Such transitions are particularly important in the motor system, specifically with respect to motor-unit recruitment and gradation of force. If we regard the electrophysiological activity of a neuron as a dynamical system, the rest-spiking transition can be thought of as a bifurcation. That is, a qualitative change in the trajectories followed by the variables in the system. Recordings from Drosophila neurons show that the spiking activity of a neuron undergoes qualitative changes as occurs after genetic manipulations that affect the population of potassium channels [1]. We developed a single compartment model of the dynamics of the identified motor neuron MN5 from Drosophila based on published experimental results [2]. In vivo, MN5 controls the dorsallongitudinal fiight muscle, firing trains of action potentials with a frequency between 6 and $25 \mathrm{~Hz}$ depending on the excitatory drive it receives. Bifurcation studies were performed to elucidate the changes in phase space as a function of different biophysical parameters including half activation potentials, gating charge, close-open rates and number of membrane channels. Bifurcation analysis and simulations predict that the number of channels and the half-activation potential for the delayed rectifiers and A-type potassium channels can explain changes in spiking behavior resulting from genetic manipulations as reported in $[1,2]$. For instance, decreasing the half activation potential for the delayed rectifier channel induces a change in bifurcation from saddle-node (no subthreshold oscillations before spiking) to Hopf (subthreshold oscillations before sustained spikes). Biophysical parameters obtained from MN5 patch clamp recordings will be used in the future to restrict the parameter space specifically for MN5. The theoretical results from this study can be tested using targeted genetic manipulations of potassium channels.

\section{Acknowledgements}

Partial support for this work was provided by the National Science Foundation IIS06 I 3404. Support for S. Berger was provided by the Interdisciplinary Graduate Program in Neuroscience.

\section{References}

I. Peng I, Wu CF: Differential contributions of shaker and shab $\mathrm{K}^{+}$currents to neuronal firing patterns in drosophila. J Neurophysiol 2007, $97: 780$.

2. Duch C, Vonhoff F, Ryglewski S: Dendrite elongation and branching are affected separately by different forms of intrinsic motoneuron excitability. J Neurophysiol 2008, 100:2525. 